\title{
Autism and ADHD - two ends of the same spectrum?
}

\author{
Włodzisław Duch, Krzysztof Dobosz, Dariusz Mikołajewski \\ Department of Informatics, Faculty of Physics, Astronomy and Informatics, \\ Nicolaus Copernicus University, ul. Grudziądzka 5, 87-100, Toruń, Poland \\ wduch@is.umk.pl
}

\begin{abstract}
Analysis of dynamics of biologically motivated neural networks allows for studying non-linear processes responsible for cognitive functions and thus provides adequate language to understand complex mental processes, including psychiatric syndromes and disorders. Problems with attention shifts that are at the roots of Autism Spectrum Disorders (ASD) and AttentionDeficit/Hyperactivity Disorder (ADHD), have been investigated using network model of Posner Visual Orienting Task (PVOT). Changing parameters that control biophysical properties of model neurons and cause network dysfunctions provides plausible explanations of many strange ASD and ADHD phenomena.
\end{abstract}

Keywords: Autism Spectrum Disorders, ASD, Attention-Deficit/Hyperactivity Disorder, ADHD, neural networks, neurodynamics, fuzzy symbolic dynamics.

\section{Introduction}

The Consortium for Neuropsychiatric Phenomics (CNP), established in 2008, aims at understanding mental disorders in a comprehensive way, comparing individual phenotypes at many levels: genetics, signaling pathways (molecular processes), structural properties of neurons, brain network responses (neuroimaging), cognitive functions, behavioral syndromes and psychiatric disorders [1]. Different disciplines contributing to neuropsychiatric phenomics, or to even more general field of neurocognitive phenomics [2], should have a common language that may be linked to behavioral, neural and molecular processes. Neurodynamics is the best candidate for such language: it may be investigated at the computational and pursued at the experimental level. Parameters of neural networks may be linked to the biophysical parameters of neurons and to the structural properties of brain networks that depend on the molecular and genetic processes. Collective states of networks investigated using neuroimaging techniques may be linked to cognitive functions and behavioral syndromes.

Understanding mental states should be based on analysis of long-time network dynamics. Multiscale brain modeling is concerned with quantitative form of electroencephalograms rather than with cognitive processes. Computational neuroscience has not yet provided general conclusions or concepts that may be of use to psychology or psychiatry. Connectionist models and cognitive architectures are constructed at too high level of abstraction to connect them with neural processes. The need for new concepts and language that will link mental states (psychology) with brain activity (neurodynamics) has been clearly stated by Spivey [3], who advocated the use of symbolic dynamics for understanding of cognitive processes. Analysis of global state

M. Lee et al. (Eds.): ICONIP 2013, Part I, LNCS 8226, pp. 623-630, 2013.

(C) Springer-Verlag Berlin Heidelberg 2013 
of the model network activity (global neurodynamics) using variety of techniques helps to uncover deeper causes of mental phenomena, providing concepts that should be useful for both psychology and neuroscience experts. In this paper we will show how relatively simple neural model of spatial attention is analyzed to provide many insights into the nature of such disorders as ASD and ADHD.

\section{Attractor Dynamics: language of mental states}

Ample theoretical and experimental evidence has proven the importance of synchronization processes in the brain. Quasi-stable patterns formed for a range of similar initial conditions are called attractors of neurodynamics. Noise drives the network out of the attractor basins, making a series of transitions between attractor states. The time spend in a given attractor basin is called dwell time. Determination of dwell time depends on the noise level in the system. The strongest source of noise is "synaptic bombardment" caused by signals coming from all over the brain to the huge dendritic trees of neurons. The landscape of attractor basins is neural network is constantly changing even without any learning, because some states become inaccessible (due to neural accommodation and other effects [4]). Emotions, sounds and other percepts change this landscape by phasic arousal, activation of key brain structures in the brain stem and limbic areas, release of major neurotransmitters.

Neurodynamics of attractor networks allows for precise discussion of temporal dynamics of mental states that is of interest to psychologist [3], and of brain processes as seen using neuroimaging techniques. Brains have a large number of regions that are interconnected, and cognitive function require cooperation of many regions. Dwell time in attractor state in each region has to be sufficiently long to enable synchronization processes that lead to global attractor states. Dwell time is estimated by clustering points of trajectory and assessing size of each cluster. Fast state transitions in some neural layers (brain regions) do not leave enough time for synchronization with other regions to occur, and therefore have no influence on global neurodynamics. Only attractor states lead to conscious mental states, enabling formation of percepts, thoughts or actions. This is stressed in the Adaptive Resonance Theory (ART) [5]. Resonant top-down attentive matching leads to synchronized activity of neurons (ART describes it at a rather high level of abstraction), and only such resonant states that are point attractors are sufficiently stable to be recognized by other parts of the brain and interpreted as conscious. Therefore procedural, spatial and motor representations that do not generate point attractors do not have qualia that are experienced in a conscious way. Although ART has been very successful in explaining a lot of data about the brain it does not use biophysically plausible neural models, and thus it is rather hard to link with molecular neuroscience.

Continuous sequences of changes responsible for recall of melodies, sentences or complex gestures and movements may be described as a special kind of "sliding attractors" that have not been studied so far, probably because they will not arise in simple low-dimensional systems that are frequently studied. 
Below the effects that lead to abnormal shifts of attention are analyzed using simple model of Posner Visual Orienting Task.

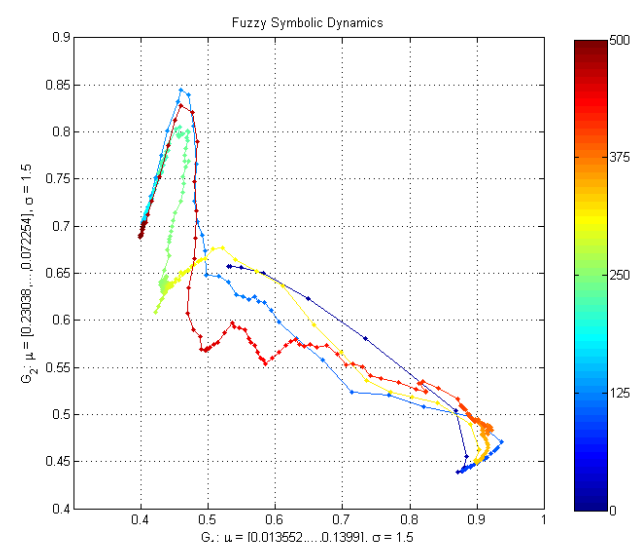

Fig. 1. Cyclic and "sliding" attractors generated by the model of motor cortex activity during repetitive movements [6], shown using Fuzzy Symbolic Dynamics technique [7,8]. In this case irregular repetitive movements have been generated, but simple movements followed by other type of actions would generate sliding attractors instead of cyclic ones. Dynamics of speech and music is full of sliding attractors.

\section{Posner Visual Orienting Task (PVOT)}

Many factors influence patterns of synchronized activation in neural networks: properties of neurons, their recurrent connections, competition and inhibition within the network that allow for multiple constraint satisfaction. The simplest models of neurons that may be linked to neurobiology should take into account at least 3 types of processes: excitatory synaptic inputs (ion channels that transport positive sodium ions outside and positive potassium ions into cell body), inhibitory synaptic inputs (ion channels that pump negative chloride ions), and leak channels that let the potassium ions to flow out. Many other ions may filter through membranes, and many type of pores or ion channels exist. The model of spatial attention presented here has been implemented in the Emergent environment [4] based on point neurons, trained by biologically-inspired mechanisms, using average rate coding, several types of neural noise, and neural accommodation (neural fatigue).

Analysis of the long-term neurodynamics cannot be reduced to statistical measures and asymptotic behavior. We have used recurrent plots and invented a new approach to visualization of high-dimensional trajectories that gives complementary information on trajectories. This approach, called Fuzzy Symbolic Dynamics (FSD) [7,8], is more informative than classical Symbolic Dynamics.

Using these tools we have investigated delays in attention shifts as a function of neural and network parameters. Although many brain areas are involved in attention control basic deficits in the ability for spontaneous attention shifts are at the core of ADHD and ASD. This fact is at the foundation of Grossberg's iSTART (imbalanced Spectrally Timed Adaptive Resonance Theory) model of autistic behaviors. In ART theory vigilance parameter controls learning of new exemplars vs. relying on already known categories of objects. New exemplars are learned when a mismatch between top-down expectation between currently active recognition category and current exemplar is detected and adaptation is not sufficient. This is a well-known idea that solves the stability-plasticity dilemma and has been used by many constructive neural 
networks that add resources where they are needed. In case of autism the iSTART model assumes hypervigillance, and thus hyperspecificity of memory, drawing many conclusions from this assumption. Such formal models give only shallow understanding: they may be fitted to the data, but give no clue what physical mechanisms are responsible for increased vigilance, how to connect such models with neuroscience, how the brain will respond to drugs. Below we shall contrast some explanations of the iSTART with our hypothesis, linking vigilance with attractors in network dynamics.

Posner Visual Orienting Test (PVOT) is perhaps the simplest experiment probing spatial attention [4]. Reaction time to brightening (or making the box lines thicker) of a small box on a screen are measured (participants press a button), and compared to the reaction time when a target box is shown in the same place (valid cue) or in a different place (invalid cue). In the neutral case typical reaction times are about 370 $\mathrm{ms}$, in the valid cue case they are about $20 \mathrm{~ms}$ shorter and in the invalid case $20 \mathrm{~ms}$ longer.

Spatial attention emerges from inhibitory competition (responsible for imposing a limitation on the total amount of activity within each region of neurons with recurrent connections), and constraint satisfaction operating throughout the network (which determines the representations that will become active in a given context). This type of attention is thus a ubiquitous property of the cortex that may be modulated by goal-

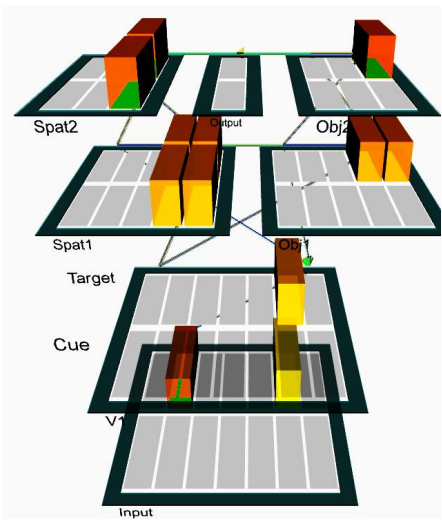
oriented control processes, but for spontaneous focus of attention a simpler model is sufficient. O'Reilly and Munakata [4] have investigated the lesions of Posner model and were able to replicate slowing reaction times of hemispatial neglect patients for targets that appear in the neglected side of space, particularly when invalidly cued. The network structure is shown here. The dorsal visual streams responsible for spatial localization represented by two layers in the left upper part (Spat1 and Spat2), and the ventral object recognition stream also by two layers (Obj1 and $\mathrm{Obj} 2)$. The spatial and object layers are connected, with stronger influence of the location on object recognition. These top layers provide increasingly spatially invariant representations, receiving inputs from the Cue/Target spatially mapped feature array that represents early visual activations (V1). The top-down influence of spatial activation after presentation of the cue weakly inhibits other locations in the input and influences object recognition. Stimuli activate one of the 7 distinct regions of the one-dimensional input array, the lower row position of the cue stimulus and the upper row the target. The cue appears at the input layer and is copied to V1, its position is recognized at the Spat2 level, and it is identified by the Obj2 layer in an invariant way. Each Spat and Obj unit represents 3 adjacent locations in the V1 layer. The Output units activate when the system settles (activity of object units exceeds 0.6 ) and is used to measure reaction times.

In case of autism or ADHD there are no lesions, but there may be many changes in the strength of connections and in neural properties. In the language of neurodynam- 
ics spontaneous trapping of attention is due to strong attractors, patterns that are difficult to break. This strength of a given attractor may be studied by initializing the system in the attractor basin, adding different amount of noise and observing the dwell time before transition is made. Changes in various network and neuron control model parameters may lead to similar behaviors. To avoid too many graphs and tables only qualitative summary is given below.

- Relative strength of the influence of spatial attention on object recognition (Spat $=>\mathrm{Obj}$ ) reduced to zero makes neutral and valid trial times identical, but leaves the $20 \mathrm{~ms}$ difference between valid and invalid cases (top-down modulation effect). Increase of this relative strength leads to slow increase of all reaction times but the $20 \mathrm{~ms}$ differences are fairly stable between $1-5$, with tendency to increase the invalid/neutral difference to $30 \mathrm{~ms}$ and slightly decrease valid/neutral trials difference.

- Decrease of relative strength of the influence of V1 layer on parietal spatial attention areas leads to sharp increase in the invalid case, attention remains fixed for a longer time on the cue. Decrease of this parameter by half (from 2 to 1) increases the time difference between neutral and invalid trials 3 times. This may be one of the contributing factors to the problems with attention shifts in autism. While local circuits are well developed there is some evidence that distal connections are weak and functional connections in autism have been linked to a variant of MET gene that shows high expression in the occipital cortex [9].

- Relative strength of recurrent connections in Spat1 and Spat2 layers has no influence on valid trials, weak influence on neutral, but stronger local connections significantly increase reaction times of invalid trials. This mechanism may also contribute to long delays in shifts of attention. TSC gene can cause local overconnectivity in the sensory cortices (visual, auditory) reducing normal neuronal pruning.

- Self-regulatory dynamics of neurons depends on complex processes, changing conductance of the ion channels (voltage-dependent gates). Changing time constants for increases in intracellular calcium that builds up slowly as function of activation in all neurons has big influence on all reaction times, reducing the difference between all types of trials to zero and making reactions for valid trials slower than for invalid and neutral. These processes depend on many types of ion channels and thus many genes are implicated.

- Increase of maximal conductance for excitatory channels (mostly glutamatergic synaptic sodium channels) above 1 leads to sharp two-fold increase in invalid trial reaction times, and small decrease of the normal/valid trials reaction times; decrease of this parameter slows down reaction times but keeps the differences roughly constant.

- Increasing maximal conductance for inhibitory channels quickly increases the invalid trials reaction times without much change in results for other trials; decrease has relatively small effect.

- Parameter regulating maximal conductance of leak (potassium $\mathrm{K}^{+}$) channels changed from 0.001 to 1.3 has relatively small influence on reaction times, but be- 
yond this value all reaction times become much longer and similar to each other. Strong leak currents decrease membrane potentials and activation of neurons takes longer time. The KCNK the two-pore-domain potassium leak channels are the main suspect in this case.

- Noise may be included either as the variance of the value of membrane potential, or variance of the synaptic input. The first type of noise makes the switch from invalid cue to the target position faster, decreasing sharply the time for invalid trials and to a smaller degree also other times. Attractors become weaker and transitions may be made faster. Synaptic noise has the opposite effect, competition between competing patterns becomes stronger and achieving the threshold for decision takes longer. High density of synapses will contribute to the "synaptic bombardment" type of noise.

The network used in Posner experiments is too simple to show full range of attractor network behavior. However, these conclusions have been confirmed by more complex models of normal reading and dyslexia, and of simple repetitive movements $[6,10]$ implemented also in the Emergent simulator. The first of these models [10] has 6 layers, representing information about orthography (6x8 units), phonology (14x14 units) and semantics (10x14 units), connected to each other via intermediate (hidden) layers of neurons. Full connectivity between each adjacent layer is assumed, with recurrent self-connections within each of these layers. The qualitative dependence of the spontaneous shifts of attention has been analyzed calculating dwell times, confirming results of the PVOT study described above. Because of the lack of space the motor cortex model is not described here [6].

Accommodation (neural fatigue) has strong influence on synchronization. Lack of accommodation with strong excitations and recurrent connections enables creation of persistent patterns, or forming deep basins of attraction with small variance, trapping neurodynamics for a long time. Attention remains focused on one concept for unusually long time, as it happens in autism spectrum disorders (ASD). Strong accommodation leads to weak synchronization, shallow basins of attractors, rapid jumps from one basin to another, with short dwell times. Such behavior is typical in case of Attention Deficit Hyperactivity Disorder (ADHD).

\section{Conclusions: towards neuropsychiatric phenomics}

This short overview of some of the mechanisms that may contribute to deregulation of attention shows that ADHD and ASD disorders should form a continuum, with typical adults displaying some autistic or ADHD traits. Different types of neurons, connections, regions of the brain may be affected as a result of multiple etiologies, including metabolic and immune system deregulation, or prenatal and early childhood exposure to various drugs, chemicals, pollutants and stressful environmental factors.

There are over 20 theories of autism spectrum disorders [13], including minicolumnopathy, mirror neuron system (MNS), underconnectivity theory, empathizingsystemizing theory, executive dysfunction theory, function over/under-connectivity theories. Most of these theories mistake symptoms for real causes. Attention is at the 
foundation for development of all cognitive functions. Developmental perspective is needed to understand how deep basins of attraction in localized sensory cortex may lead to impoverished stimulation of the brain with complex percepts, the underconnectivity of distal connections, poor development of the theory of mind and mirror system network, lack of disengagement by the default mode network. Increased number of genomic research results in huge genomic data bases, and together with other phenotypes gives hope for more precise diagnosis that will cluster similar ASD cases. Allen Human Brain Atlas of gene expression data contains 63 candidate genes (449 variants) that have been implicated in autism, and 292 genes that contribute to "other neurodegenerative disease" (as of August, 2013). Various perturbations of neural properties, at the synaptic, membrane and general connectivity level, may lead to very similar behavioral symptoms disturbing neural synchronization. Brain atlas helps to find links between gene mutations, regions in which expression occurs, results of computational simulations and likely disorders that results from such damage to the cell structure. Many such links have been identified in this article. Strong expression in occipital lobe (except occipital pole) of normal subjects is noted for MET, KCNS1, NRXN1, SLC6A10P, NPY1R genes. It has already been suggested that MET has influence on neural circuits relevant to the processing of complex visual stimuli, including faces. KCNS gene family coding two-pore delayed-rectifier potassium voltage-gated ion channels, that has strong expression in sensory areas, fits very well to our prediction that the leak channels may be responsible for slowing down in ASD or speeding up attention shifts in ADHD. Thus although these two disease have never been linked at least some subtypes may result from different mutations of the same genes that damage specific leak channels in a different way.

Following consequences of deep attractor basins are expected:

- deficits in attention disengagement;

- overspecific memory for images, words, numbers, facts, movements, except for impaired memory for olfaction [14], because many types of neural receptors important in olfaction are dysfunctional in ASD and ADHD;

- strong focus on single stimulus, easy absorption, possible sensory overstimulation by weak signals trapping the network in deep attractor, while stimuli of the same modality, like loud alarming sounds or visual signals, are ignored;

- in motor cortex repetitive cyclic sequential attractors or precise sliding attractors;

- problems with generalization and associations;

- integration of perception from different modalities are impaired, resulting in underconnectivity between distant brain areas;

- echolalia, repeating words without understanding (no associations);

- nouns will be acquired more readily than abstract words and verbs;

- fast changing stimuli, including faces, may be ignored because time is needed to escape from current deep attractor state, gaze will tend to focus on simple stimuli;

- play will be schematic, play with other children avoided in favor of simple toys.

Is it possible to compensate for some ASD dysfunctions by training the network, will it lead to faster attention shifts? Perhaps slow broadening of attractor basins will be a good strategy, or using transcranial magnetic stimulation to add noise that will 
push the network out of deep attractor. In case of ADHD psychostimulants such as Methylphenidate are used. This may be surprising but shallow basins of attractors are made deeper (neural synchronization becomes stronger) as a result of higher activation, and stimulants reduce reuptake of dopamine and norepinephrine, and thus increase activation of excitatory receptors.

There is a great need for comprehensive theories that can provide common language in the space between genetics, molecular biology and behavioral psychiatry. The development of computational models of attention, visual recognition, simple movements, and associations of concepts, models that elucidate mechanisms leading to symptoms of mental disorders, is at the very center of neuropsychiatric phenomics.

Acknowledgements. This research was supported by the Polish Ministry of Science and National Education (MNiSW/N519 5781 38).

\section{References}

1. Consortium for Neuropsychiatric Phenomics: http://www.phenomics.ucla.edu

2. Duch W.: Brains and Education: Towards Neurocognitive Phenomics. In: Learning while we are connected, Eds. N. Reynolds, M. Webb, M.M. Sysło, V. Dagiene. Vol. 3, pp. 12-23

3. Spivey M.: The continuity of mind. New York: OUP (2007)

4. O'Reilly R.C, Munakata Y.: Computational Explorations in Cognitive Neuroscience Understanding the Mind by Simulating the Brain. MIT Press, Cambridge (2000); Emergent Neural Network Simulation System http://grey.colorado.edu/emergent/

5. Grossberg, S.: Neural Dynamics of Autistic Behaviors: Learning, Recognition, Attention, Emotion, Timing, and Social Cognition. In: The Comprehensive Guide to Autism. V. B. Patel, V. R. Preedy, and C. R. Martin, Eds. Springer, London (2012)

6. Dobosz K, Mikołajewski D, Wójcik G.M, Duch W.: Simple Cyclic Movements as a Distinct Autism Feature-Computational Approach. J. of Computer Science (in print, 2013)

7. Dobosz K, Duch W.: Understanding neurodynamical systems via Fuzzy Symbolic Dynamics. Neural Networks 23, 487-496 (2010)

8. Duch W, Dobosz K.: Visualization for understanding of neurodynamical systems. Cognitive Neurodynamics 5(2), 145-160 (2011)

9. Judson M.C, Eagleson K.L, Levitt P.: A new synaptic player leading to autism risk: Met receptor tyrosine kinase. J. of Neurodevelopmental Disorders 3(3), 282-292 (2011)

10. Duch W, Nowak W, Meller J, Osiński G, Dobosz K, Mikołajewski D, Wójcik G.M.: Computational approach to understanding autism spectrum disorders. Computer Science 13(2): 47-61 (2012)

11. Just M.A, Keller T.A, Malave V.L, Kana R.K, Varma S.: Autism as a neural systems disorder: A theory of frontal-posterior underconnectivity. Neuroscience and Biobehavioral Reviews 36, 1292-1313 (2012)

12. Rudie, J.D. et al.: Autism-Associated Promoter Variant in MET Impacts Functional and Structural Brain Networks. Neuron 75(5), 904-915 (2012)

13. Zimmerman A.W. (Ed.) Autism: Current theories and evidence. Humana Press (2008)

14. Galle SA, Courchesne V, Mottron L, Frasnelli J.: Olfaction in the autism spectrum. Perception. 42(3), 341-355 (2013) 